\title{
Convergence to Compact Sets of Inexact Orbits of Nonexpansive Mappings in Banach and Metric Spaces
}

\author{
Evgeniy Pustylnik, Simeon Reich, and Alexander J. Zaslavski \\ Department of Mathematics, The Technion-Israel Institute of Technology, 32000 Haifa, Israel \\ Correspondence should be addressed to Simeon Reich, sreich@techunix.technion.ac.il \\ Received 27 September 2008; Accepted 17 November 2008 \\ Recommended by Brailey Sims
}

We study the influence of computational errors on the convergence to compact sets of orbits of nonexpansive mappings in Banach and metric spaces. We first establish a convergence theorem assuming that the computational errors are summable and then provide examples which show that the summability of errors is necessary for convergence.

Copyright (C) 2008 Evgeniy Pustylnik et al. This is an open access article distributed under the Creative Commons Attribution License, which permits unrestricted use, distribution, and reproduction in any medium, provided the original work is properly cited.

\section{Introduction}

Convergence analysis of iterations of nonexpansive mappings in Banach and metric spaces is a central topic in nonlinear functional analysis. It began with the classical Banach theorem [1] on the existence of a unique fixed point for a strict contraction. Banach's celebrated result also yields convergence of iterates to the unique fixed point. There are several generalizations of Banach's fixed point theorem which show that the convergence of iterates holds for larger classes of nonexpansive mappings. For instance, Rakotch [2] introduced the class of contractive mappings and showed that their iterates also converged to their unique fixed point.

In view of these results and their numerous applications, it is natural to ask if convergence of the iterates of nonexpansive mappings will be preserved in the presence of computational errors. In [3], we provide affirmative answers to this question. Related results can be found, for example, in [4,5]. More precisely, in [3] we show that if all exact iterates of a given nonexpansive mapping converge (to fixed points), then this convergence continues to hold for inexact orbits with summable errors. In [6], we continued to study the influence of computational errors on the convergence of iterates of nonexpansive mappings in both Banach and metric spaces. We show there that if all the orbits of a nonexpansive 
self-mapping of a metric space $X$ converge to some closed subset $F$ of $X$, then all inexact orbits with summable errors also converge to this attractor set $F$. On the other hand, we also construct examples which show that inexact orbits may fail to converge if the errors are not summable.

Our purpose in the present paper is to consider the case where different exact orbits converge to possibly different compact subsets of X. In Section 2, we obtain a convergence result (see Theorem 2.1 below) under the assumption that the computational errors are summable. This result is an extension of [3, Theorem 4.2]. In Sections 3 and 4, we provide examples which show that the summability of errors is necessary for convergence (see Proposition 3.1 and Theorem 4.1).

\section{Convergence to compact sets}

Let $(X, \rho)$ be a complete metric space. For each $x \in X$, and each nonempty and closed subset $A \subset X$, put

$$
\rho(x, A)=\inf \{\rho(x, y): y \in A\}
$$

For each mapping $T: X \rightarrow X$, set $T^{0} x=x$ for all $x \in X$.

Theorem 2.1. Let $T: X \rightarrow X$ satisfy

$$
\rho(T x, T y) \leq \rho(x, y) \quad \forall x, y \in X
$$

Suppose that for each $x \in X$, there exists a nonempty compact set $E(x) \subset X$, such that

$$
\lim _{i \rightarrow \infty} \rho\left(T^{i} x, E(x)\right)=0 .
$$

Assume that $\left\{\gamma_{n}\right\}_{n=0}^{\infty} \subset(0, \infty), \sum_{n=0}^{\infty} \gamma_{n}<\infty$,

$$
\left\{x_{n}\right\}_{n=0}^{\infty} \subset X, \quad \rho\left(x_{n+1}, T x_{n}\right) \leq \gamma_{n}, \quad n=0,1, \ldots
$$

Then, there exists a nonempty compact subset $F$ of $X$ such that

$$
\lim _{n \rightarrow \infty} \rho\left(x_{n}, F\right)=0
$$

Proof. In order to prove the theorem, it is sufficient to show that any subsequence of $\left\{x_{n}\right\}_{n=0}^{\infty}$ has a convergent subsequence.

To see this, it is sufficient to show that for any $\epsilon>0$, the following assertion holds:

(P1) any subsequence of $\left\{x_{n}\right\}_{n=0}^{\infty}$ has a subsequence which is contained in a ball of radius $\epsilon$.

Indeed, there is an integer $k \geq 1$ such that

$$
\sum_{i=k}^{\infty} r_{i}<\frac{\epsilon}{8}
$$


Evgeniy Pustylnik et al.

Define a sequence $\left\{y_{i}\right\}_{i=k}^{\infty}$ by

$$
\begin{gathered}
y_{k}=x_{k} \\
y_{i+1}=T y_{i} \quad \text { for all integers } i \geq k
\end{gathered}
$$

There exists a nonempty compact set $E \subset X$ such that

$$
\lim _{i \rightarrow \infty} \rho\left(y_{i}, E\right)=0
$$

By (2.4), (2.7), and (2.8),

$$
\rho\left(x_{k+1}, y_{k+1}\right) \leq \gamma_{k} .
$$

Assume that $q \geq k+1$ is an integer and that for $i=k+1, \ldots, q$,

$$
\rho\left(x_{i}, y_{i}\right) \leq \sum_{j=k}^{i-1} r_{j}
$$

(Note that in view of (2.10), inequality (2.11) is valid when $q=k+1$.)

By (2.2) and (2.11),

$$
\rho\left(T y_{q}, T x_{q}\right) \leq \rho\left(y_{q}, x_{q}\right) \leq \sum_{j=k}^{q-1} r_{j}
$$

When combined with (2.4), this implies that

$$
\rho\left(x_{q+1}, y_{q+1}\right) \leq \rho\left(x_{q+1}, T x_{q}\right)+\rho\left(T x_{q}, T y_{q}\right) \leq r_{q}+\sum_{j=k}^{q-1} r_{j}=\sum_{j=k}^{q} r_{j},
$$

so that (2.11) also holds for $i=q+1$. Thus, we have shown that for all integers $q \geq k+1$,

$$
\rho\left(y_{q}, x_{q}\right) \leq \sum_{j=k}^{q-1} r_{j}<\sum_{j=k}^{\infty} r_{j}<\frac{\epsilon}{8}
$$

by (2.6). In view of (2.9), we have for all large enough natural numbers $q$,

$$
\rho\left(x_{q}, E\right)<\frac{\epsilon}{4}
$$

By (2.15), there exist an integer $q_{0}>k$ and a sequence $\left\{z_{i}\right\}_{i=q_{0}}^{\infty} \subset E$ such that

$$
\rho\left(x_{i}, z_{i}\right)<\frac{\epsilon}{3} \text { for all integers } i \geq q_{0}
$$


Consider any subsequence $\left\{x_{q_{i}}\right\}_{i=1}^{\infty}$ of $\left\{x_{n}\right\}_{n=0}^{\infty}$. Since the set $E$ is compact, the sequence $\left\{z_{q_{i}}\right\}_{i=1}^{\infty}$ has a convergent subsequence $\left\{z_{q_{i}}\right\}_{j=1}^{\infty}$.

We may assume without loss of generality that all elements of this convergent subsequence belong to $B(u, \epsilon / 16)$ for some $u \in X$.

In view of (2.16),

$$
x_{q_{i_{j}}} \in B\left(u, \frac{\epsilon}{2}\right) \text { for all sufficiently large natural numbers } j \text {. }
$$

Thus, (P1) holds and this completes the proof of Theorem 2.1.

Note that Theorem 2.1 is an extension of the following result established in [3].

Theorem 2.2. Let $(X, \rho)$ be a complete metric space and let $T: X \rightarrow X$ be such that

$$
\rho(T x, T y) \leq \rho(x, y) \quad \forall x, y \in X
$$

and for each $x \in X$, the sequence $\left\{T^{n} x\right\}_{n=1}^{\infty}$ converges in $(X, \rho)$.

Assume that $\left\{\gamma_{n}\right\}_{n=0}^{\infty} \subset(0, \infty)$ satisfies $\sum_{n=0}^{\infty} \gamma_{n}<\infty$, and that a sequence $\left\{x_{n}\right\}_{n=0}^{\infty} \subset X$ satisfies $\rho\left(x_{n+1}, T x_{n}\right) \leq \gamma_{n}, n=0,1, \ldots$ Then, the sequence $\left\{x_{n}\right\}_{n=1}^{\infty}$ converges to a fixed point of $T$ in $(X, \rho)$.

\section{First example of nonconvergence to compact sets}

In this section, we show that both Theorems 2.1 and 2.2 cannot, in general, be improved (cf. [6, Proposition 3.1]).

Proposition 3.1. For any normed space $X$, there exists an operator $T: X \rightarrow X$ such that $\| T x-$ $T y\|\leq\| x-y \|$ for all $x, y \in X$, the sequence $\left\{T^{n} x\right\}_{n=1}^{\infty}$ converges for each $x \in X$ and, for any sequence of positive numbers $\left\{\gamma_{n}\right\}_{n=0}^{\infty}$, there exists a sequence $\left\{x_{n}\right\}_{n=0}^{\infty} \subset X$ with $\left\|x_{n+1}-T x_{n}\right\| \leq \gamma_{n}$ for all nonnegative integers $n$, which converges to a compact set if and only if the sequence $\left\{\gamma_{n}\right\}_{n=0}^{\infty}$ is summable, that is, $\sum_{n=0}^{\infty} \gamma_{n}<\infty$.

Proof. This is a simple fact because we may take $T$ to be the identity operator: $T x=x, \forall x$. Then, we may take $x_{0}$ to be an arbitrary element of $X$ with $\left\|x_{0}\right\|=1$ and define by induction

$$
x_{n+1}=T x_{n}+\gamma_{n} x_{0}, \quad n=0,1,2, \ldots
$$

Evidently, $\left\|x_{n+1}-T x_{n}\right\|=\gamma_{n}$ and $x_{n+1}=x_{0}\left(1+\sum_{i=0}^{n} \gamma_{i}\right)$ for all integers $n \geq 0$, so that the convergence of $\left\{x_{n}\right\}_{n=0}^{\infty}$ to a compact set is equivalent to the summability of the sequence $\left\{\gamma_{n}\right\}_{n=0}^{\infty}$. Proposition 3.1 is proved.

\section{Second example of nonconvergence to compact sets}

In Section 3, we have shown that Theorems 2.1 and 2.2 cannot, in general, be improved. However, in Proposition 3.1 every point of the space is a fixed point of the operator $T$, and the inexact orbits tend to infinity. In this section, we construct an operator $T$ on a certain complete metric space $X$ (a bounded, closed, and convex subset of a Banach space) such that all of its 
orbits converge to its unique fixed point, and for any nonsummable sequence of errors and any initial point, there exists an inexact orbit which does not converge to any compact set (cf. [6, Theorem 4.1]).

Let $X$ be the set of all sequences $x=\left\{x_{i}\right\}_{i=1}^{\infty}$ of nonnegative numbers such that $\sum_{i=1}^{\infty} x_{i} \leq 1$. For $x=\left\{x_{i}\right\}_{i=1}^{\infty}$ and $y=\left\{y_{i}\right\}_{i=1}^{\infty}$ in $X$, set

$$
\rho\left(\left\{x_{i}\right\}_{i=1}^{\infty},\left\{y_{i}\right\}_{i=1}^{\infty}\right)=\sum_{i=1}^{\infty}\left|x_{i}-y_{i}\right|
$$

Clearly, $(X, \rho)$ is a complete metric space.

Define a mapping $T: X \rightarrow X$ as follows:

$$
T\left(\left\{x_{i}\right\}_{i=1}^{\infty}\right)=\left(x_{2}, x_{3}, \ldots, x_{i}, \ldots\right), \quad\left\{x_{i}\right\}_{i=1}^{\infty} \in X
$$

In other words, for any $\left\{x_{i}\right\}_{i=1}^{\infty} \in X$,

$$
T\left(\left\{x_{i}\right\}_{i=1}^{\infty}\right)=\left\{y_{i}\right\}_{i=1}^{\infty} \text {, where } y_{i}=x_{i+1} \text { for all integers } i \geq 1 \text {. }
$$

Set $T^{0} x=x$ for all $x \in X$. Clearly,

$$
\begin{gathered}
\rho(T x, T y) \leq \rho(x, y) \quad \forall x, y \in X, \\
T^{n} x \text { converges to }(0,0, \ldots, \ldots) \text { as } n \longrightarrow \infty
\end{gathered}
$$

for all $x \in X$.

Theorem 4.1. Let $\left\{r_{i}\right\}_{i=0}^{\infty} \subset[0, \infty)$,

$$
\sum_{i=0}^{\infty} r_{i}=\infty
$$

and $x=\left\{x_{i}\right\}_{i=1}^{\infty} \in X$. Then, there exists a sequence $\left\{y^{(i)}\right\}_{i=0}^{\infty} \subset X$ such that

$$
y^{(0)}=x, \quad \rho\left(T y^{(i)}, y^{(i+1)}\right) \leq r_{i}, \quad i=0,1, \ldots,
$$

and the following property holds.

There is no nonempty compact set $E \subset X$ such that

$$
\lim _{i \rightarrow \infty} \rho\left(y^{(i)}, E\right)=0
$$

In the proof of this theorem, we may assume without loss of generality that

$$
r_{i} \leq 16^{-1} \text { for all integers } i \geq 0 \text {. }
$$

We precede the proof of Theorem 4.1 with the following lemma. 
Lemma 4.2. Let $z^{(0)}=\left\{z_{i}^{(0)}\right\}_{i=1}^{\infty} \in X$, let $k \geq 0$ be an integer, and let $j_{0}$ be a natural number. Then, there exist an integer $n \geq 4$ and a sequence $\left\{z^{(i)}\right\}_{i=0}^{n} \subset X$ such that

$$
\begin{gathered}
\rho\left(z^{(i+1)}, T z^{(i)}\right) \leq r_{k+i}, \quad i=0, \ldots, n-1, \\
z^{(n)}=\left(z_{1}^{(n)}, \ldots, z_{i}^{(n)}, \ldots\right)=\left\{z_{i}^{(n)}\right\}_{i=1}^{\infty}
\end{gathered}
$$

with $z_{j_{0}+1}^{(n)} \geq 4^{-1}$.

Proof. There is a natural number $m>4$ such that

$$
\begin{gathered}
m>j_{0}+4, \\
\sum_{i=m}^{\infty} z_{i}^{(0)}<16^{-1} .
\end{gathered}
$$

Set

$$
z^{(i+1)}=T z^{(i)}, \quad i=0, \ldots, m-1
$$

Then,

$$
z^{(m)}=\left(z_{m+1}^{(0)}, z_{m+2}^{(0)}, \ldots, z_{i}^{(0)}, \ldots\right)
$$

By (4.5), there is a natural number $n>m$ such that

$$
\sum_{j=k+m}^{k+n} r_{j} \geq 2^{-1}
$$

By (4.14) and (4.8),

$$
n \geq m+7,
$$

and we may assume without loss of generality that

$$
\sum_{j=k+m}^{k+n-1} r_{j}<\frac{1}{2} .
$$

In view of (4.14) and (4.8),

$$
\sum_{j=k+m}^{k+n-1} r_{j}=\sum_{j=k+m}^{k+n} r_{j}-r_{k+n} \geq 2^{-1}-16^{-1} .
$$


Evgeniy Pustylnik et al.

For $i=m+1, \ldots, n$, define $z^{(i)}=\left\{z_{j}^{(i)}\right\}_{j=1}^{\infty}$ as follows:

$$
\begin{gathered}
z_{j}^{(i)}=z_{j+i^{\prime}}^{(0)} \quad j \in\{1,2, \ldots\} \backslash\left\{n+1+j_{0}-i\right\}, \\
z_{n+1+j_{0}-i}^{(i)}=z_{n+1+j_{0}}^{(0)}+\sum_{j=k+m}^{k+i-1} r_{j} .
\end{gathered}
$$

Clearly, for $i=m+1, \ldots, n, z^{(i)}$ is well defined and by (4.18), (4.19), (4.10), and (4.16),

$$
\sum_{j=1}^{\infty} z_{j}^{(i)}=\sum_{j=i+1}^{\infty} z_{j}^{(0)}+\sum_{j=k+m}^{k+i-1} r_{j} \leq \sum_{j=m}^{\infty} z_{j}^{(0)}+\sum_{j=k+m}^{k+n-1} r_{j} \leq 16^{-1}+2^{-1}<1
$$

Thus $z^{(i)} \in X, i=m+1, \ldots, n$.

Let $i \in\{m, \ldots, n-1\}$. We now estimate $\rho\left(z^{(i+1)}, T z^{(i)}\right)$. If $i=m$, then by (4.2), (4.3), (4.13), and (4.18),

$$
\rho\left(z^{(i+1)}, T z^{(i)}\right) \leq r_{k+i}
$$

Let $i>m$. We first set

$$
\left\{\tilde{z}_{j}\right\}_{j=1}^{\infty}=T z^{(i)}
$$

In view of (4.14), (4.2), and (4.3), $\tilde{z}_{j}=z_{j+1}^{(i)}$ for all integers $j \geq 1$. When combined with (4.18), this implies that

$$
\begin{gathered}
\tilde{z}_{j}=z_{j+1+i}^{(0)} \quad \forall j \in\{1,2, \ldots\} \backslash\left\{n-i+j_{0}\right\} \\
\tilde{z}_{n+j_{0}-i}=z_{n+1+j_{0}-i}^{(i)}=z_{n+1+j_{0}}^{(0)}+\sum_{j=k+m}^{k+i-1} r_{j} .
\end{gathered}
$$

By (4.18) and (4.23),

$$
\tilde{z}_{j}=z_{j}^{(i+1)}
$$

for all $j \in\{1,2, \ldots\} \backslash\left\{n+j_{0}-i\right\}$. It now follows from (4.22), (4.25), (4.18), (4.19), and (4.23) that

$$
\begin{aligned}
\rho\left(z^{(i+1)}, T z^{(i)}\right) & =\rho\left(z^{(i+1)},\left\{\tilde{z}_{j}\right\}_{j=1}^{\infty}\right)=\left|z_{n+j_{0}-i}^{(i+1)}-\tilde{z}_{n+j_{0}-i}\right| \\
& =\left|z_{n+1+j_{0}}^{(0)}+\sum_{j=k+m}^{k+i} r_{j}-\left(z_{n+1+j_{0}}^{(0)}+\sum_{j=k+m}^{k+i-1} r_{j}\right)\right|<r_{k+i} .
\end{aligned}
$$


When combined with (4.12), this implies that

$$
\rho\left(z^{(i+1)}, T z^{(i)}\right) \leq r_{k+i}, \quad i=0, \ldots, n-1 .
$$

By (4.17) and (4.18),

$$
z_{j_{0}+1}^{(n)}=z_{n+1+j_{0}-n}^{(n)} \geq \sum_{j=k+m}^{k+n-1} r_{j} \geq 4^{-1}
$$

This completes the proof of Lemma 4.2.

Proof of Theorem 4.1. In order to prove the theorem, we construct by induction, using Lemma 4.2 , a sequence of nonnegative integers $\left\{s_{k}\right\}_{k=0}^{\infty}$ and a sequence $\left\{y^{(i)}\right\}_{i=0}^{\infty} \subset X$ such that

$$
\begin{gathered}
y^{(0)}=x, \\
\rho\left(y^{(i+1)}, T y^{(i)}\right) \leq r_{i} \text { for all integers } i \geq 0, \\
s_{0}=0, \quad s_{k}<s_{k+1} \text { for all integers } k \geq 0,
\end{gathered}
$$

and for all integers $k \geq 1$,

$$
y_{k+1}^{\left(s_{k}\right)} \geq \frac{1}{4}
$$

In the sequel, we use the notation $y^{(i)}=\left\{y_{j}^{(i)}\right\}_{j=1}^{\infty}, i=0,1, \ldots$

Set

$$
y^{(0)}=x, \quad s_{0}=0
$$

Assume that $q \geq 0$ is an integer and that we have already defined a (finite) sequence of nonnegative numbers $\left\{s_{k}\right\}_{k=0}^{q}$ and a (finite) sequence of points $\left\{y^{(i)}\right\}_{i=0}^{s_{q}} \subset X$ such that (4.33) is valid, (4.30) holds for all integers $i$ satisfying $0 \leq i<s_{q}$,

$$
s_{i}<s_{i+1} \text { for all integers } i \text { satisfying } 0 \leq i<q \text {, }
$$

and that (4.32) holds for all integers $k$ satisfying $0<k \leq q$. (Note that for $q=0$ this assumption does hold.)

Now, we show that this assumption also holds for $q+1$.

Indeed, applying Lemma 4.2 with

$$
z^{(0)}=y^{\left(s_{q}\right)}, \quad j_{0}=q+1, \quad k=s_{q},
$$


we obtain that there exist an integer $s_{q+1} \geq 4+s_{q}$ and a sequence $\left\{y^{(i)}\right\}_{i=s_{q}}^{s_{q+1}} \subset X$ such that

$$
\begin{gathered}
\rho\left(y^{(i+1)}, T y^{(i)}\right) \leq r_{i}, \quad i=s_{q}, \ldots, s_{q+1}-1, \\
y_{q+2}^{\left(s_{q+1}\right)} \geq \frac{1}{4} .
\end{gathered}
$$

Thus, the assumption made for $q$ also holds for $q+1$. Therefore, we have constructed by induction a sequence of points $\left\{y^{(i)}\right\}_{i=0}^{\infty} \subset X$ and a sequence of nonnegative integers $\left\{s_{k}\right\}_{k=0}^{\infty}$ which satisfy (4.30) and (4.31) for all integers $i, k \geq 0$, respectively, and (4.32) for all integers $k \geq 1$.

Finally, we show that there is no nonempty compact set $E \subset X$ such that

$$
\lim _{i \rightarrow \infty} \rho\left(y^{(i)}, E\right)=0
$$

Assume the contrary. Then, there does exist a nonempty compact set $E \subset X$ such that

$$
\lim _{i \rightarrow \infty} \rho\left(y^{i)}, E\right)=0
$$

This implies that any subsequence of $\left\{y^{(k)}\right\}_{k=0}^{\infty}$ has a convergent subsequence.

Consider such a subsequence $\left\{y^{\left(s_{q}\right)}\right\}_{q=1}^{\infty}$. This subsequence has a convergent subsequence $\left\{y^{s_{q p}}\right\}_{p=1}^{\infty}$. There are therefore a point $z=\left\{z_{i}\right\}_{i=0}^{\infty} \in X$ such that

$$
z=\lim _{p \rightarrow \infty} y^{\left(s_{q_{p}}\right)}
$$

and a natural number $p_{0}$ such that

$$
\rho\left(z, y^{\left(s_{q p}\right)}\right) \leq 16^{-1} \text { for all integers } p \geq p_{0} .
$$

Hence we have, for all integers $p \geq p_{0}$,

$$
\begin{gathered}
\left|z_{q_{p}+1}-y_{q_{p}+1}^{\left(s_{q_{p}}\right)}\right| \leq \rho\left(z, y^{\left(s_{q p}\right)}\right) \leq 16^{-1}, \\
z_{q_{p}+1} \geq y_{q_{p}+1}^{\left(s_{q_{p}}\right)}-16^{-1}>8^{-1} .
\end{gathered}
$$

This, of course, contradicts the inequality $\sum_{i=1}^{\infty} z_{i} \leq 1$. The contradiction we have reached completes the proof of Theorem 4.1.

\section{Acknowledgments}

This research was supported by the Israel Science Foundation (Grant no. 647/07), the Fund for the Promotion of Research at the Technion, and by the Technion President's Research Fund. 


\section{References}

[1] S. Banach, "Sur les opérations dans les ensembles abstraits et leur application aux équations intégrales," Fundamenta Mathematicae, vol. 3, pp. 133-181, 1922.

[2] E. Rakotch, "A note on contractive mappings," Proceedings of the American Mathematical Society, vol. 13, no. 3, pp. 459-465, 1962.

[3] D. Butnariu, S. Reich, and A. J. Zaslavski, "Convergence to fixed points of inexact orbits of Bregmanmonotone and of nonexpansive operators in Banach spaces," in Fixed Point Theory and Its Applications, pp. 11-32, Yokohama, Yokohama, Japan, 2006.

[4] D. Butnariu, S. Reich, and A. J. Zaslavski, "Asymptotic behavior of inexact orbits for a class of operators in complete metric spaces," Journal of Applied Analysis, vol. 13, no. 1, pp. 1-11, 2007.

[5] A. M. Ostrowski, "The round-off stability of iterations," Zeitschrift für Angewandte Mathematik und Mechanik, vol. 47, no. 2, pp. 77-81, 1967.

[6] E. Pustylnik, S. Reich, and A. J. Zaslavski, “Inexact orbits of nonexpansive mappings," Taiwanese Journal of Mathematics, vol. 12, no. 6, pp. 1511-1523, 2008. 